\title{
Pensonomonoor
}

2017, vol. 78, 168-178

http://dx.doi.org/10.12657/denbio.078.016

\author{
Teresa Hazubska-Przybyt, Mikołaj Wawrzyniak
}

\section{Stimulation of somatic embryo growth and development in Picea spp. by polyethylene glycol}

Received: 21 March 2017; Accepted: 13 September 2017

\begin{abstract}
The objective of the presented study was to compare the effect of osmotic stress, induced by various concentrations of polyethylene glycol (PEG) MW 4000, on the development, maturation, and conversion of somatic embryos of Picea abies (model species) and P. omorika (endemic species) into somatic seedlings. Embryogenic tissue (ET) of $P$. abies (line A and B) and $P$. omorika were incubated on a maturation medium with $20 \mu \mathrm{M}$ abscisic acid (ABA), $1 \mu \mathrm{M}$ indole-3-butyric acid (IBA) and PEG 4000 at 0, 5, and 7.5\%. After 5 weeks, the number of somatic embryos produced, and the number of cotyledonary somatic embryos per 1 gram of ET, was assessed. Cotyledonary somatic embryos were selected and transferred to a Margara germination medium for two months. Their growth, as measured by hypocotyl and radicle length, was evaluated after one month, and the somatic embryos were transferred to fresh medium. After the second month of incubation, the conversion frequency of germinated embryos into the seedlings was assessed. In general, ETs of both of the tested spruce species cultured on a maturation medium amended with $5 \%$ PEG 4000 exhibited a tendency to produce a higher total number of embryos, as well as a higher number of cotyledonary embryos. The B line of $P$. abies produced a significantly greater number of cotyledonary embryos, relative to the control (no PEG). PEG only stimulated hypocotyl growth in embryos of both lines (A and B) of $P$. abies during the germination stage. Somatic embryos of Picea omorika were characterized by poor growth of both hypocotyls and radicles, irrespective of PEG treatment. The addition of PEG 4000 to the maturation medium did not improve the conversion frequency of germinated somatic embryos into somatic seedlings in either spruce species after a month of incubation on Margara medium. The effect of PEG 4000 on somatic embryogenesis efficiency was more evident in the ET of the two $P$. abies lines, relative to the $P$. omorika ET line, during both the maturation and the germination stage. Results indicated that the $P$. omorika ET line was almost completely insensitive to all of the PEG concentrations tested.
\end{abstract}

Keywords: somatic embryogenesis, osmotic stress, germination, Norway spruce, Serbian spruce

Address: M. Wawrzyniak, T. Hazubska-Przybył, Institute of Dendrology, Polish Academy of Science, Parkowa 5, 62-035 Kórnik, e-mail: hazubska@o2.pl

\section{Introduction}

Somatic embryogenesis (SE) is a biological process in which somatic cells develop into embryos without going through a reproductive process involving fertilization and zygote development. Although SE occurs primarily under in vitro conditions, it has also been reported to occur in nature in Kalanchoë (Fehér, 2006). Utilizing SE to propagate plants is an efficient process that allows for the production 
of large numbers of morphologically and genetically identical embryos from a small number of explants. In addition, SE also avoids the impact of many environmental and biological factors such as, climate, erratic seed production, periodicities in flowering etc., which can have a major impact on embryo development (Lelu-Walter et al., 2013; Smertenko \& Bozhkov, 2014). Moreover, SE can be used to produce genetically-modified plants and synthetic seeds. Combined with various cryopreservation technologies, SE also enables the preservation of valuable or endangered genotypes of plants ex situ (Aquea et al., 2008; Ma et al., 2012). Although many conifers (e.g. Abies, Larix, Pinus, Picea, and Pseudotsuga) exhibit SE (Chen et al., 2010) there are still problems associated with the induction of SE and the production of somatic seedlings. For example, it is still very difficult to produce embryogenic tissue (ET) from vegetative tissues of mature conifers, thus the process can be very time consuming, costly, and inefficient. Therefore, immature zygotic embryos isolated from seeds are typically the source of explants used for the induction of SE in conifers (Klimaszewska \& Cyr, 2002). The ability to obtain zygotic embryos, however, is limited each year to the short period of time associated with seed development (Lara-Chavez et al., 2011; Merkle et al., 2014). The ability to induce SE in trees is strongly dependent on the genotype of the explants, while the proper selection of specific PGRs, their combination and concentrations, have a lesser effect on the induction of SE (Shoji et al., 2006; Maruyama \& Hosoi, 2012).

The appropriate course of development during the early stages of the SE process is essential in order for somatic embryo development to occur. In turn, the correct maturation of somatic embryos determines their ability to develop into seedlings. Thus, this stage of SE is key to the successful production of plants using this method of micropropagation (Pullman et al., 2003a; Montalbán et al., 2010; Merkle et al., 2014).

The addition of abscisic acid (ABA) to the growth medium during the initial stages of the SE process triggers developmental changes that enable embryogenic tissue to form somatic embryos. Subsequent to the formation of mature, somatic embryos, however, only a low percentage of them are converted into somatic seedlings (Stasolla et al., 2002). Improvement in the quality of somatic embryos and their further development into somatic seedlings can be achieved by exposing ETs and SEs to osmotic stress, which plays an important role in regulating embryo development, both in vitro and in vivo (Bozhkov \& von Arnold, 1998; Belmonte et al., 2005). Osmotic stress in cultures of conifer tissues can be induced by the use of several compounds, such as amino acids, mannitol, or dextran. The addition of polyethylene glycol (PEG) to the maturation medium, however, has become the most common method used to stimulate embryo maturation (Belmonte et al., 2005; Heringer et al., 2013; Hudec et al., 2016). PEG is a non-permeating osmoticum that increases desiccation tolerance and induces the deposition of storage proteins in cells (Attree et al., 1991; Misra et al., 1993; Heringer et al., 2013). The positive effect of PEG on embryo maturation has been reported for many coniferous species, including Picea abies (Hudec et al., 2016), P. likiangensis (Chen et al., 2010), P. glauca (Stasolla et al., 2002; Stasolla \& Yeung, 2003), and Cryptomeria japonica (Maruyama et al., 2007). Hosoi and Maruyama (2012) reported a 25-fold increase in the number of mature embryos produced in Pinus luchuensis, relative to the number of mature embryos produced in PEG-free medium when embryogenic tissue was incubated on a medium containing 15\% PEG. Tret'yakova and Barsukova (2012) reported that somatic embryos of Larix sukaczewii only developed and matured on a medium supplemented with $10 \%$ PEG. Substituting 7.5\% PEG for maltose in a maturation medium resulted in faster somatic embryo development in Pinus taeda and the production of large, bullet-shaped embryos (Pullman et al., 2003b).

The development of ET into mature, somatic embryos is the initial step required for the production of healthy somatic seedlings with well-developed and functional root and shoot systems (Hay \& Charest, 1999). The development of both a shoot apical meristem (SAM) and a root apical meristem (RAM) in a somatic embryo is a crucial stage in the production of somatic seedlings. SAM and RAM formation is essential to both somatic embryo germination and further development, since seedling growth is dependent upon both shoot and root emergence. The quality of somatic embryos can be assessed by monitoring the development of shoot meristems (Kong \& Yeung, 1992). PEG treatment has been reported to contribute to proper SAM formation and to affect the regulation of embryonic root development in Picea glauca (Stasolla \& Yeung, 2003). The authors demonstrated that exposure of somatic embryos to PEG resulted in higher transcript abundance of genes responsible for the control of organ development and for SAM and RAM formation in somatic embryos. A higher abundance of transcripts for these genes indicates that PEG promoted the induction and development of shoots and roots in somatic embryos.

The aim of the present study was to compare the effect of osmotic stress induced by various concentrations of PEG 4000 on the maturation and germination of Picea abies and P. omorika somatic embryos and their conversion into somatic seedlings. Picea abies is a widely-distributed spruce species in Eurasian forests and is a model species in studies on $\mathrm{SE}$, while $P$. omorika is an endemic and relict Balkan species, cultivated in Europe as an ornamental tree. In contrast to $P$. abies, $P$. omorika is able to grow in a 
variety of climatic and soil conditions, and is tolerant to the pollution often found in urban environments (Leljak-Levanić et al., 2009). Due to its overall tolerance to abiotic stress, this spruce species has the potential to be used to solve many problems connected with deforestation in the coniferous forests of Central Europe (Kolevska-Pletikapić \& Buturović-Derić, 1995). Many protocols have been studied and reported for the induction of SE in P. abies, however, SE induction in P. omorika has been rarely studied. Budimir and Vujičić (1992), first reported the induction of SE in P. omorika from seedling explants. Mature zygotic embryos have also been successfully used as explants in P. omorika (Kolevska-Pleticapić et al., 1995). Only a few protocols have been reported for somatic embryo maturation in P. omorika and their further development into somatic seedlings (Vujičić \& Budimir, 1995; Tramisak-Milaković et al., 1999; Hazubska \& Szczygieł, 2003; Szczygieł et al., 2007; Hazubska-Przybył \& Bojarczuk, 2008). Therefore, further research is needed on the induction of SE and subsequent somatic seedling development in this spruce species, in order to ensure that this endemic, relict tree species can be adequately preserved and propagated.

\section{Methods}

\section{Plant material}

Somatic embryos were obtained from embryogenic tissues (ETs) of Norway spruce (Picea abies) and Serbian spruce (Picea omorika) derived from mature zygotic embryos isolated from mature seeds (Fig. 1A). Seeds of Norway spruce were collected from trees growing in the experimental forest of 'Zwierzyniec' near Kórnik (provenance experiment, origin of seeds - Kolonowskie) and from a single tree of Serbian spruce growing in the Kórnik Arboretum $\left(52^{\circ} 15^{\prime} \mathrm{N}, 17^{\circ} 04^{\prime} \mathrm{E}\right)$. Explants were incubated on half-strength Litvay's medium ( $1 / 2 \mathrm{LM}$ ) (Litvay et al., 1985) containing $9 \mu \mathrm{M}$ 4-amino-3,5,6-trichloropicolinic acid (Picloram), $4.5 \mu \mathrm{M}$ 6-benzyladenine (BA), sucrose (10 g/l), and Phytagel (5 g/l). The same medium was used for the proliferation of the induced ETs. Two lines of Norway spruce (A and B) and one line of Serbian spruce were used in the experiments.

\section{Experiment I: Effect of PEG concentration on somatic embryo maturation}

ET of $P$. abies and $P$. omorika were transferred to $1 / 2$ LM medium without growth regulators, supplemented with active charcoal $(10 \mathrm{~g} / \mathrm{l})$. After one week, $2 \mathrm{~g}$ of tissue were submerged in $25 \mathrm{ml}$ of liquid growth medium (LM). The culture tubes were then vigorously shaken in order to break up the aggregates of cells. Five $\mathrm{ml}$ of mixture of the medium with tissue was then transferred to filter paper (70 $\mathrm{mm}$ diameter). The remaining medium was removed from the filter paper with a small vacuum pump (KNF Laboport ${ }^{\mathrm{TM}}$ type N 820.3 FT. 18). The filter paper containing $0.4 \mathrm{~g}$ of ET was placed on Petri dish with $1 / 2$ LM medium supplemented with ABA $(20 \mu \mathrm{M})$, IBA $(1 \mu \mathrm{M})$, sucrose $(34 \mathrm{~g} / \mathrm{l})$, Phytagel $(6 \mathrm{~g} / \mathrm{l})$, and either 0 (control), 5 , or $7.5 \%$ PEG MW 4000. The placement of the tissue on the filter paper provided it with even access to the medium during the maturation process. Approximately $20 \mathrm{ml}$ of medium was used in each $90 \mathrm{~mm}$ Petri dish. The ETs were then incubated for 5 weeks at $22 \pm 1^{\circ} \mathrm{C}$ under blue-red LED lights $\left(35 \mu \mathrm{M} \mathrm{m}^{-2} \mathrm{~s}^{-1}\right)$. The number of somatic embryos produced in the cultures of each spruce species per $1 \mathrm{~g}$ of ET, as well as the number of embryos that had reached the cotyledonary stage of development, were assessed as the proportion in $0.4 \mathrm{~g}$ of ET per filter paper. The experiments conducted on two lines (A and B) of Picea abies were repeated five and three times, respectively. In the case of $P$. omorika, the experiments on the single line of $P$. omorika were repeated four times.

In conifers, PEG MW 4000 is considered to be the most beneficial molecular weight for the induction of somatic embryogenesis (Bozhkov \& von Arnold, 1998; Krajňáková et al., 2013; Pullman \& Bucalo, 2014; Merkle et al., 2014). The use of small molecular weight (200 and 400) PEG has no effect on the maturation process, most likely because it can diffuse into cells (Attree et al., 1995). In contrast, too high of a molecular weight (e.g. PEG MW 8000) PEG has an adverse impact on somatic embryo maturation (Szczygieł, 2005).

\section{Experiment II: Effect of PEG on somatic embryo germination and seedling development}

Selected somatic embryos at the cotyledonary stage (Fig. 1B) of both spruce species were placed on Margara germination medium (Margara, 1977) lacking plant growth regulators but amended with supplemental sucrose $(20 \mathrm{~g} / \mathrm{l})$. The embryos were cultured for 2 weeks at $22 \pm 1^{\circ} \mathrm{C}$ in the dark and then under red-blue LED light $\left(35 \mu \mathrm{M} \mathrm{m}^{-2} \mathrm{~s}^{-1}\right)$ for an additional two weeks. Twenty embryos were placed in one Petri dish (diameter $90 \mathrm{~mm}$ ). From 118 to 415 cotyledonary somatic embryos of both spruce species were tested depending on the ET line and treatment 

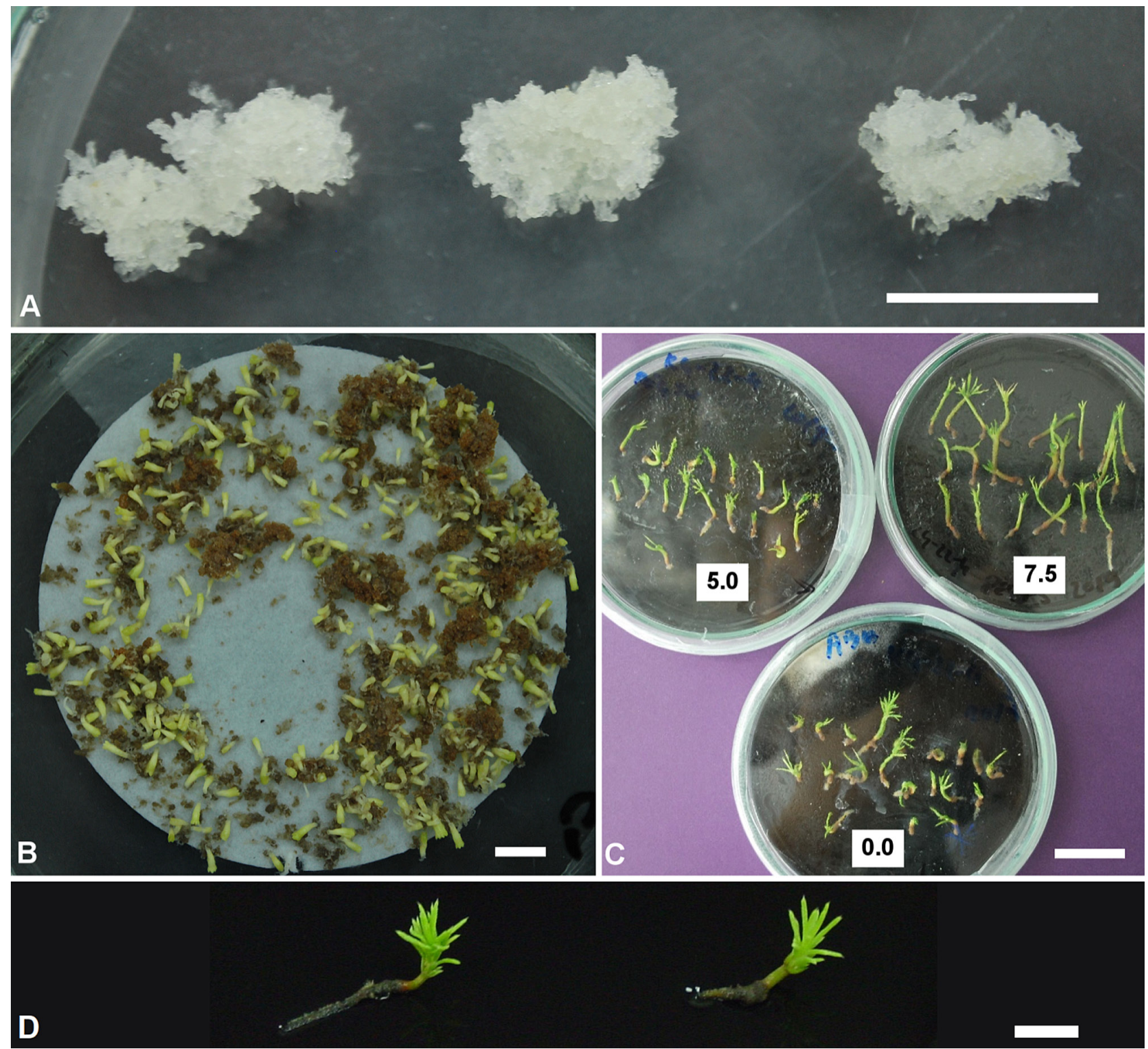

Fig. 1. A - Embryogenic tissue (ET) of Picea omorika prior to the transfer to maturation medium amended with different concentrations of PEG 4000. Bar $=10 \mathrm{~mm}$; B - Cotyledonary somatic embryos of Norway spruce (Picea abies). Bar $=5 \mathrm{~mm} ; \mathrm{C}-$ Germinated somatic embryos of Picea abies (line B) cultured on Margara medium after control or PEG treatments during the maturation stage. Bar $=10 \mathrm{~mm}$; D - functional somatic seedlings of $P$. omorika with developed radicles and epicotyls. Bar $=10 \mathrm{~mm}$

variant. After the one-month period of incubation, the frequency of germination was assessed and the growth of the somatic embryos was estimated, as measured by hypocotyl and radicle length. The embryos were subsequently cultured on the same medium for an additional month in order to evaluate their ability to be converted into somatic seedlings. Somatic embryos were considered as converted into a seedling if the length of the hypocotyl and radicle exceeded 10 and $5 \mathrm{~mm}$, respectively; and if root hairs and epicotyls had developed.

\section{Statistical analysis}

StatisticaTM 10 (Statsoft Poland) software was used for data analysis. In Experiment I and II, data were analyzed using the Tukey's HSD test at a significance level of $p<0.05$. Due to the limited amount of plant material available, the number of times the experiment was repeated varied between the lines as follows: P. abies (line A), $n=5$; P. abies (line B), $n=3$, P. omorika, $n=4$. The statistical analyses were based on the averages of the number of embryos obtained in 2-6 Petri dishes for a single replicate. A separate analysis of variance (ANOVA) was performed on the data obtained from each of the studied lines. 


\section{Results}

\section{Experiment I}

The ET lines of both spruce species varied in their ability to produce embryos. Line B of $P$. abies was the most proficient, producing 398 embryos/1 g ET in the no-PEG control (Fig. 2). A slightly lower number of embryos, 310 embryos/1 g ET was produced by $P$. omorika (Fig. 2). Line A of P. abies was the least proficient line, producing only 170 embryos/1 g ET in the no-PEG control (Fig. 2). In both species, all of the ET lines exhibited a tendency to increase the production and maturation of somatic embryos when the medium was amended with 5\% PEG 4000 (Fig. 2). The number of somatic embryos in line A of $P$. abies increased from 170 to 313 embryos/1 g ET, but the difference was not statistically significant $(p=0.0812)$. Additionally, the increased number of somatic embryos produced in the medium amended with 5\% PEG 4000, also did not result in the production of

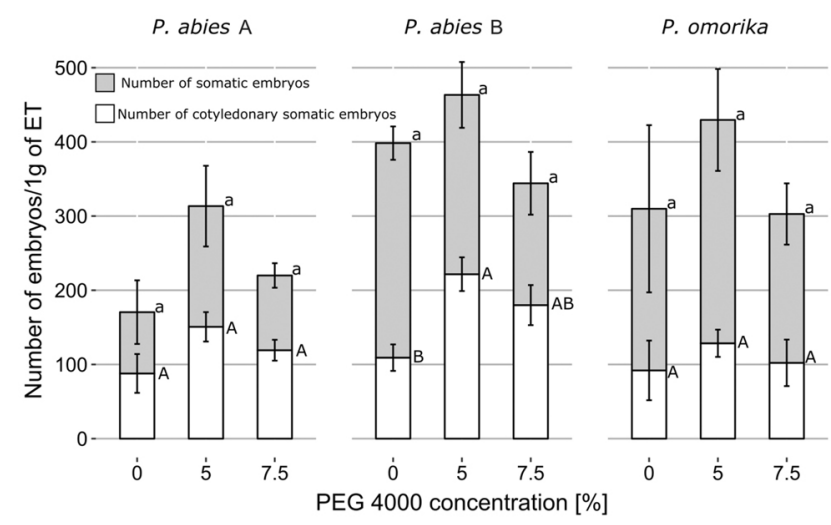

Fig. 2. Effect of various PEG 4000 concentrations within the maturation medium on the total number of embryos and cotyledonary somatic embryos produced in Picea abies ET lines A and B and P. omorika during the maturation stage. A Tukey's test was performed separately for each line. Values with different letters are significantly different at $\mathrm{p} \leq 0.05$. Mean $\pm \mathrm{SE}, \mathrm{n}=4-6$ a significantly different number of somatic embryos at the cotyledonary stage, relative to the other treatments. The number of cotyledonary somatic embryos in line A of $P$. abies was approximately $50 \%$ of the total number of embryos that were produced (Fig. 2) and this percentage was similar to the control and other PEG 4000 treatments.

Application of $7.5 \%$ PEG 4000 decreased the total number of somatic embryos, as well as the number of cotyledonary embryos, relative to the $5 \%$ PEG treatment; however, the decrease was not statistically significant. In contrast, line B was more responsive than line A to the presence of PEG in the maturation medium. The number of cotyledonary-stage embryos produced in line B was significantly different relative the no-PEG control and the 5\% PEG 4000 treatment (Fig. 2). The number of total embryos and cotyledonary embryos produced when $7.5 \%$ PEG 4000 was added to the medium was slightly lower, relative to the 5\% PEG 4000 treatment, but the number of cotyledonary-stage embryos was still higher relative to the no-PEG control (Fig. 2). Cotyledonary somatic embryos accounted for 48 and $52 \%$ of all of the embryos obtained on the medium supplemented with 5 and $7.5 \%$ PEG 4000, respectively. These levels were approximately 2 -fold higher than the control treatment where the rate was approximately $28 \%$ (Fig. 2).

In the case of $P$. omorika, the addition 5\% PEG 4000 to the maturation medium also resulted in the production of a higher number of somatic embryos (430 embryos/1 g of ET) and cotyledonary embryos relative to the no-PEG control; however, again the difference was not statistically significant (Fig. 2). The level of embryo production in the no-PEG control and the $7.5 \%$ PEG 4000 treatment was similar and not statistically significant from each other. The proportion of cotyledonary somatic embryos produced was similar in all of the tested treatments $(0,5$, and $7.5 \%$ PEG 4000), reaching approximately $30 \%$ of all of the somatic embryos produced (Fig. 2).

Table 1. Percentage of somatic embryo germination and conversion to somatic seedlings in Picea abies (lines A and B) and P. omorika cultured on maturation medium amended with 0,5 , or $7.5 \%$ PEG 4000

\begin{tabular}{cccccc}
\hline Species & Line & PEG 4000 concentration (\%) & No. of embryos on the medium & Germination (\%) & Conversion* (\%) \\
\hline & & 0 & 258 & $99.31 \pm 0.70 \mathrm{a}$ & $3.03 \pm 1.24 \mathrm{a}$ \\
& $\mathrm{A}$ & 5 & 245 & $100.00 \mathrm{a}$ & $0.00 \mathrm{a}$ \\
Picea abies & 7.5 & 240 & $100 \mathrm{a}$ & $1.06 \pm 0.36 \mathrm{a}$ \\
& & 0 & 57 & $98.33 \pm 1.67 \mathrm{a}$ & $3.51 \pm 1.76 \mathrm{a}$ \\
& $\mathrm{B}$ & 5 & 152 & $100.00 \mathrm{a}$ & $1.14 \pm 0.66 \mathrm{a}$ \\
& 7.5 & 116 & $98.41 \pm 1.59 \mathrm{a}$ & $2.38 \pm 1.38 \mathrm{a}$ \\
P. omorika & 0 & 415 & $92.55 \pm 0.7 \mathrm{a}$ & $3.41 \pm 1.78 \mathrm{a}$ \\
& 5 & 235 & $96.43 \pm 3.05 \mathrm{a}$ & $1.44 \pm 0.92 \mathrm{a}$ \\
\hline
\end{tabular}

Mean \pm SE, $n=4-6$. Different letters indicate statistically significant differences $(p<0.05)$ within a line. ${ }^{*}$ - conversion of germinated embryos into somatic seedlings was assessed after incubation of embryos on Margara medium for an additional month after assessing germination. 


\section{Experiment II}

Cotyledonary somatic embryos of both spruce species were cultured on Margara medium for one month and the germination frequency was subsequently assessed. Results indicated that germination frequency (emergence of radicles from embryos) was 92 to $100 \%$ across all of the treatments (Table 1). Hypocotyls and radicles appeared during the first two weeks of incubation in darkness. Over the subsequent few weeks under light conditions, however, hypocotyl growth was primarily observed and radicle growth was generally poor. Somatic embryos in both lines (A and B) of P. abies that had matured on medium supplemented with both 5 and 7.5\% PEG 4000 produced significantly longer hypocotyls, relative to the no-PEG control (Figs 1C and 3). The longest hypocotyls were observed in somatic embryos that had germinated on Margara medium amended with 7.5\% PEG, where average hypocotyl length was approximately $19 \mathrm{~mm}$ and $14 \mathrm{~mm}$ in lines A and B of P. abies, respectively (Fig. 3). In contrast, hypocotyl length in lines $A$ and $B$ in the no-PEG control were 13 and 6 mm, respectively (Fig. 3).

In contrast to hypocotyls, radical length in both lines of P. abies was similar or slightly shorter in all of the PEG treatments, relative to the no-PEG control (Fig. 3). Neither 5 or $7.5 \%$ PEG 4000 had any effect on hypocotyl or radical length in P. omorika. Average hypocotyl length was approximately $5 \mathrm{~mm}$ and average radicle length ranged between $2.4-3.3 \mathrm{~mm}$ in all of the tested treatments (Fig. 3). In general, hypocotyl growth was less in the one line of P. omorika in relative comparison to both lines of $P$. abies. Similar results were obtained for radicle growth. Overall, radicle and hypocotyl growth appeared to be more highly synchronized in P. omorika than in P. abies.

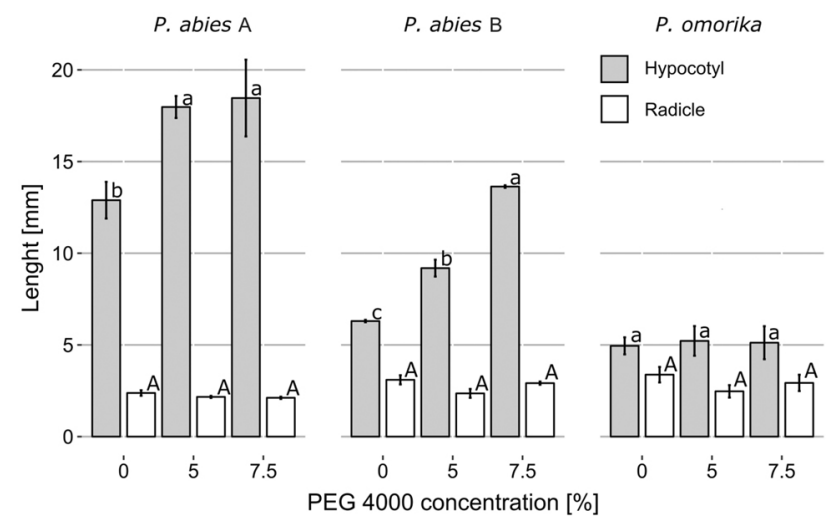

Fig. 3. Effect of 0,5 , or $7.5 \%$ PEG 4000 added to the maturation medium on germination of Picea abies (lines A and B) and P. omorika somatic embryos. A Tukey's test was performed separately for each line. Values with different letters are significantly different at $\mathrm{p} \leq 0.05$. Mean $\pm \mathrm{SE}, \mathrm{n}=4-6$
The ability to convert germinated somatic embryos into somatic seedlings was poor when the germinated somatic embryos were cultured on Margara medium for an additional month. Only as much as $3.5 \%$ and $3.4 \%$ of the P. abies and P. omorika embryos, respectively, developed radicles longer than $5 \mathrm{~mm}$ along with the initial development of root hairs and an epicotyl in the no-PEG control treatment (Table 1 and Fig. 1D). As indicated in Table 1, a low level of conversion was also observed for embryos treated with 5\% PEG 4000 (P. abies line B, $P$ omorika) and 7.5\% PEG 4000 (P. abies line A and B).

\section{Discussion}

The present study demonstrated that osmotic stress induced by PEG 4000 had a variable impact on the maturation and germination of somatic embryos of two ET lines (A and B) of P. abies. A minor effect of this osmoticum on the maturation of somatic embryos of the endemic spruce species, was also observed; however, the effect was not statistically significant. In contrast, no effect of PEG 4000 was observed on the frequency of somatic embryo germination process in any of the tested lines of either species, although the P. abies hypocotyl was significantly improved.

During the maturation step, PEG treatments resulted in a tendency to produce a higher number of embryos, relative to the no-PEG controls, in all of the ET lines; irrespective of spruce species during the maturation stage. Approximately $25 \%$ more cotyledonary somatic embryos were obtained in line A of $P$. abies when the medium was supplemented with $5 \%$ PEG 4000, although this result was not significant. In contrast, exposure of line B of P. abies to 5\% PEG 4000 had significant effect on the number of cotyledonary somatic embryos produced. Small but no significant differences were also found in the number of cotyledonary somatic embryos produced in the line of Picea omorika in response to PEG 4000. A distinct positive effect of PEG on the maturation of somatic embryos has also been reported by Attree et al. (1991). In that study, a 3-fold increase in the number of cotyledonary-stage somatic embryos of $P$. glauca produced was observed, relative to the control, when ETs were placed on a maturation medium supplemented with either 5 or $7.5 \%$ PEG. Svobodová et al. (1999) and Find (1997) have also reported a demonstrable improvement in P. abies somatic embryo quality and development when ETs were placed on a maturation medium supplemented with either 3.75 or 5\% PEG. These studies found that the maturation process in P. abies was shortened from 5 to 3-4 weeks and also noted changes in some morphological traits in PEG-treated somatic embryos. In contrast, no morphological differences between PEG-treated and 
no-PEG embryos were observed in the present study. In our study, the maturation process also lasted 5 weeks in both the no-PEG control and PEG 4000 treatments in both of the utilized spruce species. The ambiguous effect of PEG on embryo maturation in different lines of Picea abies observed in the present study are in agreement with results reported by Szczygieł (2003). In that study, improved somatic embryo quality was observed in only some of the tested lines when ETs were placed on maturation medium supplemented with PEG 3350. Krajňáková et al. (2013) also reported a differential response to PEG in different genotypes of silver fir (Abies alba). In their study, maturation medium supplemented with $10 \%$ PEG 4000 produced better quality somatic embryos. In addition, they also observed a significant increase in the number of cotyledonary somatic embryos in one line and a completely opposite result in a second line. These authors stated that a high rate of maturation was dependent on a combination of several factors, including the genotype of the cell lines and the concentration of ABA and PEG used. In the case of $P$. omorika, a general lack of comparative research exists on the application of PEG as a factor affecting somatic embryo maturation. Hazubska (2005), however, reported that PEG 3500 had no beneficial effect on the number or quality of $P$. omori$k a$ somatic embryos produced. Results obtained on the line of $P$. omorika tested in the present study agreed with this prior report. In earlier studies of $P$. omorika SE, only $12 \mu \mathrm{M}$ ABA was sufficient to stimulate somatic embryos development without the use of an osmotic agent (Budimir \& Vujičić, 1992). Additionally, our previous research has also demonstrated the potential stimulation of somatic embryo production in both spruce species by the treatment of ETs with 10-60 $\mu \mathrm{M} \mathrm{ABA}, 1 \mu \mathrm{M}$ IBA, and 3.4\% sucrose (Hazubska-Przybył \& Bojarczuk, 2008; Hazubska \& Szczygieł, 2003). Tramišak-Milaković et al. (1999) also demonstrated that 20 or $40 \mu \mathrm{M} \mathrm{ABA}$ in maturation medium supplemented with $3 \%$ glucose, sucrose, or maltose, depending on the ET line, was optimal for somatic embryo development. Higher osmotic pressure resulting from the use of a higher concentration of sugars $(6 \%)$ in the medium resulted in decreasing the production of mature embryos, relative to media supplemented with $3 \%$ of the same sugars. A similar tendency was also observed in the lines of both $P$. abies and P. omorika with the use of 7.5\% PEG 4000. Leljak-Levanić et al. (2009) also noted the importance of the concentration of PGRs in the proliferation medium on the number and morphology of $P$. omorika somatic embryos during their development on maturation medium. They found that previous exposure of ETs to a medium containing different auxin/cytokinin ratios, or a hormone-free medium supplemented with activated charcoal, improved somatic embryo maturation on subsequent ABA-containing medium. A similar effect was observed in P. abies (Bozhkov et al., 2002). This approach has also been used in our research on both spruce species, but its effectiveness was found to differ between ET lines (Hazubska \& Szczygieł, 2003). Many studies have indicated that PEG is an inducer of both somatic embryo maturation and the conversion of somatic embryos into functional seedlings (Heringer et al., 2013). Supplementing a culture medium with PEG, a non-permeating osmoticum, simulates the water stress that occurs during normal seed development and this is believed to affect somatic embryo maturation (Heringer et al., 2013; Koehler et al., 2013). In mature somatic embryos, PEG stimulates cotyledon development, the deposition of storage materials, the synthesis of endogenous $\mathrm{ABA}$, and improves desiccation tolerance (Attree et al., 1995; Find, 1997; Svobodová et al., 1999; Stasolla \& Yeung, 2003; Shoji et al., 2006). The current study revealed that supplementing maturation medium with PEG affected the growth of hypocotyls disproportionally to the growth of the radicle during the germination stage in both lines of P. abies, but had no effect on the growth of either organ in the tested line of $P$. omorika. Asynchronous development of radicles and hypocotyls has also been observed in our earlier studies on different lines of both spruce species, irrespective of the concentration of $\mathrm{ABA}$ used in the maturation medium (Hazubska-Przybył et al., 2008). We hypothesize that the variable response observed in the different lines and species is due to genetic or physiological differences in the examined ET lines. According to Park (2002), however, genetics explains only $3 \%$ of the variation observed in the rate of somatic embryo germination. Regardless, some reports have indicated that genotype has a clear impact on the results obtained in the use of PEG on somatic embryo maturation (Bozhkov \& von Arnold, 1998). Genetics appears to be the most likely reason that, in spite of the reported beneficial effect of PEG on somatic embryo development, several authors have also reported an adverse effect of PEG. Although maturation medium supplemented with PEG increases the number and quality of the somatic embryos produced, somatic embryos treated with PEG in many cases fail to germinate and develop into functional seedlings (Kong \& Yeung, 1995; Find, 1997; Bozhkov \& von Arnold, 1998). As a result, the overall benefit of exposing embryogenic lines to various concentrations of PEG in the maturation medium remains controversial (Hudec et al., 2016). Somatic embryos in many conifer species are partially dried at high relative humidity or desiccated in the presence of supersaturated solutions of salt in order to improve germination and conversion rates (Roberts et al., 1990; Jones \& van Staden, 2001; Dronne 
et al., 1997). This approach has also been used in our recent studies (Hazubska-Przybył et al., 2015) and resulted in an improvement in radicle growth in both $P$. abies and P. omorika embryos when they were exposed to partial drying at $97 \%$ relative humidity for 2 weeks. In the case of $P$. omorika, however, the extent of hypocotyl growth was insufficient for obtaining functional somatic seedlings. In contrast, Budimir and Vujičić (1992) obtained a 51.9\% rate of germination in P. omorika somatic embryos after 3 weeks of cultivation of cotyledonary stage embryos on a PGRfree germination medium, and without any additional post-maturation treatments. This approach was also indicated to be effective by Mihaljević and Jelaska (2005). We also observed high conversion rates in a few lines of $P$. omorika without the drying phase (Szczygieł et al., 2007). Our more recent experience, however, has shown that obtaining germinating somatic embryos and functional seedlings for most ET lines is still problematic due to the poor synchronization of radicle and hypocotyls development (Hazubska-Przybył et al., 2008). A 96\% germination rate in P. omorika somatic embryos was obtained when they were cultured in maturation medium supplemented with $0.8 \%$ agar (Kolevska-Pleikapić \& Buturović-Derić, 1995). This observation was the basis of examining the influence of an osmotic agent, such as PEG 4000 , on the whole process of somatic seedling development.

The highest concentration (7.5\%) of PEG $4000 \mathrm{did}$ not produce a consistent negative effect on the germination of somatic embryos in either of the examined spruce species. A slight decrease in root length was observed in both lines of P. abies, and up to a $50 \%$ increase in hypocotyl length was observed in line B of $P$. abies, relative to the no-PEG control, when somatic embryos were exposed to $7.5 \%$ PEG 4000 . All of the treatments resulted in poor growth in P. omorika, which suggests that PEG did not affect the germination of somatic embryos in this spruce species.

Germinating somatic embryos of Picea abies exhibited poor synchronization between hypocotyl and radicle growth. Excessive hypocotyl growth, relative to radical growth, was observed in both lines of $P$. abies, while weak growth of both organs was found in the line of P. omorika. After one month of incubation on Margara germination medium, the PEG 4000 treatments had no effect on the rate of conversion of somatic embryos into seedlings in either species. The conversion frequency was similar in no-PEG and PEG-treated embryos. These results stand in contrast to the report by Bozhkov and von Arnold (1998), in which PEG treatment of somatic embryos was found to suppress radicle elongation and lateral root formation, even after ex vitro transfer of the plantlets. Högberg et al. (2001) reported that the inhibitory effect of PEG on germination disappeared over time.
No significant influence of PEG on the survival or growth of somatic embryos was observed in P. abies somatic seedlings after prolonged ex vitro growth (Högberg et al., 2001).

In conclusion, the results of the present study indicate that supplementing the maturation medium with 5\% PEG 4000 resulted in greater production of cotyledonary somatic embryos in P. abies (line B). The osmotic stress induced by the PEG 4000, however, did not significantly affect the maturation process in somatic embryos of P. omorika. A tendency to produce higher numbers of total somatic embryos and cotyledonary somatic embryos in ETs of both species was observed when maturation medium was supplemented with 5\% PEG 4000 . The presence of 5 and $7.5 \%$ PEG 4000 in the maturation medium also resulted in greater hypocotyl growth, relative to the no-PEG control, in both lines of $P$. abies during the germination stage of somatic embryos but had little impact on root growth. In fact, poor synchronization between root and hypocotyl growth was observed in germinating embryos of P. abies. The addition of PEG 4000 to the maturation medium had no effect on the germination of somatic embryos in P. omorika. Additionally, PEG 4000 did not affect the conversion of germinating embryos into the somatic seedlings in either spruce species. We hypothesize that the data obtained in the present study are the result of both a genotype effect and the effect of the physiological state of the ETs. These physiological and genetic differences result in a differential response to the presence of PEG in the maturation medium. Furthermore, the physiological status of the somatic embryos during maturation and germination, as determined by the levels of endogenous growth regulators (especially ABA), as well as water content, may also play a significant role in the obtained results. Additional research on the physiological and genetic status of ETs will be required in order to better understand the reasons underlying the weak response of some ET lines of spruce (especially P. omorika) to PEG. Such studies would greatly assist in resolving the problems associated with producing high-quality, somatic seedlings in recalcitrant conifer species and lines within a species.

\section{Acknowledgments}

This work was supported by the National Science Centre (NCN) in Krakow, Poland, grant No N N309 130837).

\section{Authors' contribution}

THP: conducted the research, interpreted the results, and prepared the manuscript for publication.

MW: conducted the research, interpreted the results, and prepared the manuscript for publication. 


\section{References}

Aquea F, Poupin MJ, Matus JT, Gebauer M, Medina C \& Arce-Johnson P (2008) Synthetic seed production from somatic embryos of Pinus radiate. Biotechnological Letters 30: 1847-1852. doi:10.1007/s10529-008-9754-X.

Attree SM, Moore D, Sawhney VK \& Fowke LC (1991) Enhanced maturation and desiccation tolerance of white spruce [Picea glauca (Moench) Voss] somatic embryos: effects of a non-plasmolysing water stress and abscisic acid. Annals of Botany London 68: 519-525.

Attree SM, Pomeroy MK \& Fowke LC (1995) Development of white spruce (Picea glauca (Moench.) Voss) somatic embryos during culture with abscisic acid and osmoticum, and their tolerance to drying and frozen storage. Journal of Experimental Botany 46: 433-439. doi:10.1093/jxb/46.4.433.

Belmonte MF, Macey J, Yeung EC \& Stasolla C (2005) The effect of osmoticum on ascorbate and glutathione metabolism during white spruce (Picea glauca) somatic embryo development. Plant Physiology and Biochemistry 43: 337-346. doi:10.1016/j.plaphy.2005.01.022.

Bozhkov PV \& von Arnold S (1998) Polyethylene glycol promotes maturation but inhibits further development of Picea abies somatic embryos. Physiologia Plantarum104: 211-224. doi:10.1034/j.1399-3054.1998.1040209.x.

Bozhkov PV, Filonova L \& von Arnold S (2002) A key developmental switch during Norway spruce somatic embryogenesis is induced by withdrawal of growth regulators and is associated with cell death and extracellular acidification. Biotechnology and Bioengineering 77: 658-667.

Budimir S \& Vijičić R (1992) Benzyladenine induction of buds and somatic embryogenesis in Picea omorika (Pancić) Purk. Plant Cell Tissue and Organ Culture 31: 89-94.

Chen S, Chen S, Chen F, Wu T, Wang Y \& Yi S (2010) Somatic embryogenesis in mature zygotic embryos of Picea likiangensis. Biologia 65: 853-858. doi:10.2478/s11756-010-0089-4.

Dronne S, Label P \& Lelu MA (1997) Desiccation decreases abscisic acid content in hybrid larch (Larix $\mathrm{x}$ leptoeuropea) somatic embryos. Physiologia Plantarum 99: 433-438.

Fehér A (2006) Why somatic plant cells start to form embryos? Somatic embryogenesis. Springer, Berlin Heidelberg, pp. 85-101.

Find JI (1997) Changes in endogenous ABA levels in developing somatic embryos of Norway spruce (Picea abies (L.) Karst.) in relation to maturation medium, desiccation and germination. Plant Science 128: 75-83. doi:10.1016/S01689452(97)00141-6.
Hay EI \& Charest PJ (1999) Somatic embryo germination and desiccation tolerance in conifers: Somatic embryogenesis in woody plants. Springer, pp. 61-96.

Hazubska T (2005) Micropropagation of selected spruce species (Picea abies, P. omorika, P. pungens "Glauca" and P. breweriana) via somatic embryogenesis. Dissertation. Institute of Dendrology Polish Academy of Sciences.

Hazubska T \& Szczygiel K (2003) Induction of somatic embryogenesis in spruce: Picea omorika, $P$. pungens 'Glauca', $P$. breweriana and $P$. abies. Dendrobiology 50: 17-24.

Hazubska-Przybył T \& Bojarczuk K (2008) Somatic embryogenesis of selected spruce species (Picea abies, $P$. omorika, $P$. pungens 'Glauca' and $P$. breweriana). Acta Societatis Botanicorum Poloniae 77: 189-199.

Hazubska-Przybył T, Bojarczuk K \& Guzicka M (2008) Structure of embryogenic tissues and accumulation of storage materials in somatic embryos of Picea abies and P. omorika. Dendrobiology 60: 19-28.

Hazubska-Przybył T, Wawrzyniak M, Obarska A \& Bojarczuk K (2015) Effect of partial drying and desiccation on somatic seedling quality in Norway and Serbian spruce. Acta Physiologiae Plantarum 37: 1735.

Heringer AS, Vale EM, Barroso T, Santa-Catarina C \& Silveira V (2013) Polyethylene glycol effects on somatic embryogenesis of papaya hybrid UENF/ CALIMAN 01 seeds. Theoretical and Experimental Plant Physiology 25: 116-124.

Hosoi Y \& Maruyama E (2012) Plant regeneration from embryogenic tissue of Pinus luchuensis Mayr, an endemic species in Ryukyu Island. Plant Biotechnology 29: 401-406. doi:10.5511/plantbiotechnology.12.0530a.

Högberg KA, Bozhkov PV, Grönroos R \& von Arnold $S$ (2001) Critical factors affecting ex vitro performance of somatic embryo plants of Picea abies. Scandinavian Journal of Forest Research16: 295304. doi:10.1080/02827580116772.

Hudec L, Konràdovà $\mathrm{H}$, Haškovà $\mathrm{A}$ \& Lipavskà $\mathrm{H}$ (2016) Norway spruce embryogenesis: changes in carbohydrate profile, structural development and response to polyethylene glycol. Tree Physiology 36: 548-561. doi:10.1093/treephys/tpw016.

Jones NB \& van Staden J (2001) Improved somatic embryo production from embryogenic tissue of Pinus patula. In Vitro Cellular and Developmental Biology-Plant 37: 543-549.

Koehler AD, Carvalho CR, Abreu IS \& Clarindo WR (2013) Somatic embryogenesis from leaf explants of hermaphrodite Carica papaya: a new approach for clonal propagation. African Journal of Biotechnology 12: 2386-2391. doi:10.5897/AJB12.1939. 
Kolevska-Pletikapić B \& Buturović-Derić Z (1995) Regeneration of Picea omorika via organogenesis. Plant Cell Tissue and Organ Culture 41: 189-192.

Kolevska-Pletikapić B, Krsnik-Rasol M, Lorković Z, Besendorfer V, Ramišak T \& Jelaska S (1995) Somatic embryogenesis in Picea omorika (Panč.) Purk. Acta Pharmaceutica 45: 267-271.

Klimaszewska K \& Cyr DR (2002) Conifer somatic embryogenesis: I. Development. Dendrobiology 48: 31-39.

Kong L \& Yeung EC (1992) Development of white spruce somatic embryos: II. Continual shoot meristem development during germination. In Vitro Plant 28: 125-131. doi:10.1007/BF02823060.

Kong L \& Yeung EC (1995) Effects of silver nitrate and polyethylene glycol on white spruce (Picea glauca) somatic embryo development: enhancing cotyledonary embryo formation and endogenous ABA content. Physiologia Plantarum 93: 298304. doi:10.1111/j.1399-3054.1995.tb02232.x.

Krajňáková J, Bertolini A, Gömöry D, Vianello A \& Häggman H (2013) Initiation, long-term cryopreservation, and recovery of Abies alba Mill. embryogenic cell lines. In Vitro Cellular and Developmental Biology-Plant 49: 560-571. doi:10.1007/ s11627-013-9512-1.

Lara-Chavez A, Flinn BS \& Egertsdotter U (2011) Initiation of somatic embryogenesis from immature zygotic embryos of Oocarpa pine (Pinus oocarpa Schiede ex Schlectendal). Tree Physiology 31: 539-554. doi:10.1093/treephys/tpr040.

Leljak-Levanić D, Mihaljević S \& Jelaska S (2009) Variations in DNA methylation in Picea omorika (Panč) Purk. embryogenic tissue and the ability for embryo maturation. Propagation of Ornamental Plants 9: 3-9.

Lelu-Walter MA, Thompson D, Harvengt L, Sanchez L, Toribio M \& Pâques LE (2013) Somatic embryogenesis in forestry with a focus on Europe: state-of-the-art, benefits, challenges and future direction. Tree Genetic and Genomes 9: 883-899. doi:10.1007/s11295-013-0620-1.

Litvay JD, Verma DC \& Johnson MA (1985) Influence of a loblolly pine (Pinus taeda L.). Culture medium and its components on growth and somatic embryogenesis of the wild carrot (Daucus carota L.). Plant Cell Reports 4: 325-328. doi:10.1007/ BF00269890.

Ma X, Bucalo K, Determann RO, Cruse-Sanders JM \& Pullman GS (2012) Somatic embryogenesis, plant regeneration, and cryopreservation for Torreya taxifolia, a highly endangered coniferous species. In Vitro Cellular and Developmental Biology - Plant 48: 324-334. doi:10.1007/s11627-012-9433-4.

Margara J (1977) La multiplication vegetative de la betterave (Beta vulgaris L.) en culture in vitro.
ComptesRendus de l'Académie des Sciences 285: 1041-1044.

Maruyama TE \& Hosoi Y (2012) Post-maturation treatment improves and synchronizes somatic embryo germination of three species of Japanese pines. Plant Cell Tissue and Organ Culture 110: 45-52. doi:10.1007/s11240-012-0128-7.

Maruyama E, Hosoi Y \& Ishii K (2007) Polyethylene glycol enhance somatic embryo production in Japanese cedar (Cryptomeria japonica D. Don). Propagation of Ornamental Plants 7: 57-61.

Mihaljević S \& Jelaska S (2005) Omorika spruce (Picea omorika): Protocol for somatic embryogenesis in woody plants (ed. by SM Jain \& PK Gupta) Springer, Netherlands, pp. 35-45.

Merkle SA, Montello PM, Reece HM \& Kong L (2014) Somatic embryogenesis and cryostorage of eastern hemlock and Carolina hemlock for conservation and restoration. Trees 28: 1767-1776. doi:10.1007/s00468-014-1084-0.

Misra S, Attree SM, Leal I \& Fowke LC (1993) Effect of abscisic acid, osmoticum, and desiccation on synthesis of storage proteins during the development of white spruce somatic embryos. Annals of Botany London 71: 11-22. doi:10.1006/ anbo.1993.1002.

Montalbán IA, Diego ND \& Moncaleán P (2010) Bottlenecks in Pinus radiata somatic embryogenesis: improving maturation and germination. Trees 24: 1061-1071. doi:10.1007/s00468-010-0477-y.

Park YS (2002) Implementation of conifer somatic embryogenesis in clonal forestry: technical requirements and deployment considerations. Annals of Forestry Sciences 59: 651-656.

Pullman GS, Montello P, Cairney J, Xu N \& Feng X (2003a) Loblolly pine (Pinus taeda L.) somatic embryogenesis: maturation improvements by metal analyses of zygotic and somatic embryos. Plant Science 164: 955-969. doi:10.1016/S01689452(03)00079-7.

Pullman GS, Johnson S, Peter G, Cairney J \& Xu N (2003b) Improving loblolly pine somatic embryo maturation: comparison of somatic and zygotic embryo morphology, germination, and gene expression. Plant Cell Reports 21: 747-758. doi:10.1007/s00299-003-0586-9.

Pullman GS \& Bucalo K (2014) Pine somatic embryogenesis: analyses of seed tissue and medium to improve protocol development. New Forests 45: 353-377. doi:10.1007/s11056-014-9407-y.

Roberts DR, Sutton BCS \& Flinn BS (1990) Synchronous and high frequency germination of interior spruce somatic embryos following partial drying at high relative humidity. Canadian Journal of Botany 68: 1080-1090.

Shoji M, Sato H, Nakagawa R, Funada R, Kubo T \& Ogita S (2006) Influence of osmotic pressure 
on somatic embryo maturation in Pinus densiflora. Journal of Forest Research 11: 449-453. doi:10.1007/s10310-006-0227-6.

Smertenko A \& Bozhkov PV (2014) Somatic embryogenesis: life and death processes during apicalbasal patterning. Journal of Experimental Botany 65: 1343-1360. doi:10.1093/jxb/eru005.

Stasolla C, Kong L, Yeung EC \& Thorpe TA (2002) Maturation of somatic embryos in conifers: morphogenesis, physiology, biochemistry, and molecular biology. In Vitro Cellular and Developmental Biology - Plant 38: 93-105. doi:10.1104/pp. 015214.

Stasolla C \& Yeung EC (2003) Recent advances in conifer somatic embryogenesis: improving somatic embryo quality. Plant Cell, Tissue and Organ Culture 74: 15-35. doi:10.1023/A:1023345803336.

Szczygieł K (2003) Somatic embryogenesis of Norway Spruce (Picea abies Karst.), Scots pine (Abies alba Mill.) and European Larch (Larix decidua Mill.). Dissertation. Forest Research Institute.

Szczygieł K, Hazubska-Przybył T \& Bojarczuk K (2007) Somatic embryogenesis of selected conif- erous tree species of the genera Picea, Abies and Larix. Acta Societatis Botanicorum Poloniae 76: 7-15.

Svobodová H, Albrechtová J, Kumstỳřová L, Lipavská H, Vágner M \& Vondráková Z (1999) Somatic embryogenesis in Norway spruce: anatomical study of embryo development and influence of polyethylene glycol on maturation process. Plant Physiology and Biochemistry 37: 209-221. doi:10.1016/ S0981-9428(99)80036-9.

Tramisak-Milaković T, Mihaljević S \& Jelaska S (1999) Effects of abscisic acid and carbohydrates on the maturation of Picea omorika (Panč.) Purk. somatic embryos. Acta Botanica Croatica 58: 87-97.

Tret'yakova IN \& Barsukova AV (2012) Somatic embryogenesis in in vitro culture of three larch species. Russian Journal of Developmental Biology 43: 353-361. doi:10.1134/S1062360412060082.

Vujičić R \& Budimir S (1995) Somatic embryogenesis and plant regeneration in Picea omorika: Somatic embryogenesis in woody plants 3 (ed. by $S$ Jain, PK Gupta \& RJ Newton). Kluwer Academic Publishers, Netherlands, pp. 81-97. 